\title{
SmartFit: A Step Count Based Mobile Application for Engagement in Physical Activities
}

\author{
Atifa Sarwar*, Hamid Mukhtar*, Maajid Maqbool* and Djamel Belaid ${ }^{\dagger}$ \\ *National University of Sciences and Technology (NUST), \\ Islamabad, 44000, Pakistan \\ $\dagger$ Institut Mines-Telecom; Telecom SudParis; \\ CNRS UMR SAMOVAR 9 rue Charles Fourier, \\ 91011 Evry, France
}

\begin{abstract}
Research has found that relatively few people engage in regular exercise or other physical activities. Despite the availability of numerous mobile applications and specialized devices for self-tracking, people mostly lack the motivation for performing physical activities. In this article we present SmartFit, a mobile application that uses step count for promoting the physical activities in adults. This article points out that while considering walk, activity duration is not sufficient for determining users activeness state. Step count is another factor that should be taken into account. For this we propose an approach for converting the steps into duration for which activity has been performed. This duration is then used in Smartfit for categorizing user into different activeness levels. Gamification techniques have been incorporated in SmartFit as they are found to serve the purpose of motivating and encouraging the user. Gamification is used for awarding/deducting points to user in order to keep them engaged for longer period. Furthermore feedback is also provided to users depending upon their goal and achieved progress. The objective is to facilitate and motivate the user and then keep them engaged in carrying out the recommended level of physical activities.
\end{abstract} Count

Keywords-Feedback, Gamification, Physical Activities, Step

\section{INTRODUCTION}

As much as the human body is complex, so is the issue of human health. Currently obesity due to sedentary life style is one of the major concerns of developing nations. Sedentary life style has negative impacts on health resulting in chronic diseases such as increased risk of weight gain, metabolic disruption and premature mortality [1]. One of the growing body of research suggests that taking short breaks during sedentary behavior and performing some physical activity has positive effects on health [2]. On the other hand, there have been numerous evidences that exercise is such a factor that contributes heavily to a better health and consequently to the quality of life as well.

In general, participating in physical activity is beneficial to people of all ages. Physical activity contributes to fitness, a state in which people's health characteristics and behaviors enhance the quality of their lives. When it comes to mental health, the benefits of exercise cannot be overemphasized. A clinical study conducted by Babyak et al. [3] has revealed that treating depression with exercise was just as effective as medication, and vice versa. According to center for Disease
Control and Prevention (CDC) ${ }^{1}$, regular physical activity helps improve the overall health and fitness, and reduces the risk for many chronic diseases [4].

In the current age of technology, smart phones due to their ubiquitous nature are commonly used as a medium for promoting physical activities. But people still rely less on gadgets, mobiles phones, and other specialized devices to track a health indicator. This is in contrast to the fact that recently there has been an increase in the healthcare-related research and unprecedented growth in the systems, devices, and applications for healthcare management, be it on a personal or a larger scale. So what is the reason that despite the availability of plethora of mobile phone applications and devices, half of the people still track their health status in their head? This is due to the fact that people mostly lack the motivation for performing the physical activities. Users use these applications and ultimately lose their interest after using it for a few times only. Thus creating the eagerness of living a healthy life by engaging users, a goal that is more challenging and demands more effort.

Having outlined the problem and our motivation for solving the problem, we now describe our goals as following. We aim to use gamification in the health systems and study its impact on the user's motivation and long term engagement. For this, we propose a mobile application, SmartFit that promotes physical activities by counting user's steps and converting these steps into activity duration. To make Smartfit engaging and a source of motivation for users, gamification is incorporated in it. Gamification is defined as use of gaming elements in nongaming context [5]. McGonigal [6] in her book "Reality is Broken" writes that games have the power to satisfy human needs as well as solve real world problems. Franck in [7] suggests that gamification is one of the feature that could possibly change user's behavior and helps to develop habit as it serves the purpose of motivating the users and keep them engaged. Futhermore [8] states that games are not only used for entertainment but it also serves as a source of inspiration, persuasion and engagement. Thus in SmartFit, we have defined a scoring system that awards/deducts user's points depending on his/her progress. Points are awarded to users if their performance is up to the mark while points are deducted if they are lagging behind. So the user enjoys using the system rather then being compelled to use it unwillingly.

Unlike existing systems, which are rigid in setting the goals

${ }^{1}$ http://www.cdc.gov/ 
and fails to provide continuous motivation to the users, our proposed system is flexible and with the use of gamification techniques it provides continuous motivation to users. This help users not only in achieving the activeness goal once but also maintaining activeness afterwards.

The rest of this article is organized as following. First we briefly review existing work in Section II. Section III presents our proposed solution for converting the step count into activity duration. We describe our application, SmartFit, and present some of its key features in section IV. In section V we discuss the scoring system developed in SmartFit for user motivation. After that we show results of our proposed approach in section VI and finally conclude this article in section VII.

\section{RELATED WORK}

Recently, there has been much focus on auto-analytics for self-tracking, particularly health tracking. Several Personal Informatics systems have been developed that can help the user by facilitating the collection of personal information and the reflection on that information at any time [9][10]. However, the challenging part is to get access to user's data without overburdening the user, i.e., such information must be obtained in a non-intrusive manner. Fortunately, with the advances in mobile phone technologies, many of the recent mobile phones comes with a large number of sensors that can be used for automatic tracking of user's data without user's involvement. SitCoach [11] was designed for creating sedentary awareness among users. Using the built-in accelerometer in the smart phone, the user's activity is classified in an active or inactive state. Whenever a certain number of sedentary minutes is recorded, SitCoach reminds the user to take a break from sitting. Reminders are given in the form of audio and tactile messages. Similarly FitBit+ [12] aims to decrease sedentary behavior by detecting when people have been inactive for longer period and then generate messages for taking short breaks and perform some physical activity. User wears device known as Fitbit that transmits data related to steps taken, intensity of physical activity, duration of activity, distance etc. to their workstation. This data is analyzed and if user is found inactive for longer period, the alerts are generated prompting them to perform some physical activity. However, as we have identified, activeness is not just the prevention of the sedentary behavior, but inculcating the eagerness of living an active life; a goal that is more challenging and requires more treatment. The Motivate [13] project is about the design of a smartphone application that promotes the adoption of a healthy and active lifestyle. It provides personalized and contextualized advice based on geo information, weather, user location and agenda. However, the context parameters used have nothing to do with user's overall goal of activeness. In fact, the user has no fitness goal whatsoever, and hence may not find the application useful at all after giving it a try a few times only.

StepUp [14] is a mobile application that uses sensor enabled mobile phones to count the number of steps taken by a user. The aim of StepUp is to provide users a quantitative measure of their daily activities and facilitate them to integrate regular exercise into their daily life. StepUp built its own algorithm for counting the number of steps taken by user. However, StepUp only provide users with the information of the activities performed by them and does not include any feature that keep users engaged towards the application.
Players are usually addicted to video games and they prefer to play video games rather than playing outdoor games. This can cause obesity or other chronic diseases. Hirzallah [15] aims to incorporate exercise in the video games so to make games a source of healthy life. The paper targets games where players virtually live inside some city, forest or a war zone. A treadmill design has been proposed that act as a device to capture player's commands for movements. These movements include running, walking, stopping and turning. In this way the players enjoy exercising as well as playing the game. However, sooner or later, the players get exhausted due to exercise and force them to exit the game. That way, such a proposal would keep players healthy. But this design requires a separate device for playing the games as well as extra space is required for the proposed treadmill. With the emergence of more friendly consoles like Microsoft Xbox, this problem will be a serious concern.

Sedentaware [16] is a mobile application that relies on activity recognition to identify sedentary users. Users can set their goals and recommendations are given to them for completing goals. User's activity is monitored using Android API and feedback is provided in the form of alerts to prompt user for performing some physical activity. However Sedentaware lacks in producing a continuous source of motivation to use it for longer period. Users can set their goals but no approach has been defined for awarding or penalizing the users if they fail to complete their goals. This will ultimately lose user's interest and they may not find it helpful in developing a habit of living an active life.

Step Up Life [17] is a smart phone application that encourages users to have a healthy life style by performing contextually suitable physical exercises. Step Up Life provides physical activity reminders to the user after detecting a prolonged interval of sitting. Reminders are provided by considering the user's context information like user location, personal preferences, time of the day and the weather. This makes Step Up Life smart enough to provide timely and relevant feedback. However an approach can be defined for awarding the users on performing exercise when reminder appears. This will create a feeling of accomplishment in the user which in turn results in high motivation.

Authors in [18] have developed Phone Row, a smartphone boat racing game that requires users to make rowing movements and therefore engage in moderate-intensity physical activity. With these rowing movements, users can control the movement of a virtual boat across a virtual track on an external screen. However, the authors reported that due to sub-optimal implementation, users would not want to replay the game and thus not developing a habit of performing a physical activity. Move2Play [19] uses sensor enabled smart phones to measure, assess and recommends physical activity. The focus of Move2Play is on the number of steps taken by the user as well as on walking distance. For this purpose a pedometer has been built in the application. Goals are set at the start but can be customized lately according to the needs. Users are motivated by showing progress, providing rewards, publishing scores to the social network and by associating emotions to the avatar. Users are awarded with points when their performance is up to mark but no points deduction is considered when they lag behind in completing their goal. This will ultimately lose user's interest and fail to engage users for longer period.

As identified above, the existing systems do not provide 
continuous motivation to the users and thus fail to keep them engaged for longer period. Due to lack of motivation, users cannot develop a habit to make exercise, a vital component of their life. User uses these systems and will ultimately give up after giving it a few tries. SmartFit engages users by not only providing feedback but also provides the flexibility to change their goals whenever required. Use of gamification techniques for awarding points on performing activities and deducting points on not performing any activity will further boost user's emotions to use the system as a part of their daily life activities. Furthermore as per our knowledge no such system exists that make use of step count to calculate the duration for which the activity has performed. This feature makes our proposed solution distinct from the existing systems and opens new doors of thoughts towards health systems.

\section{From Step Count to ACtivity Duration}

We consider a physical activity as any activity performed by the user that involves significant movement of the various parts of the body. According to the WHO physical activity recommendations [20], one needs to do two types of physical activity each week to improve health - aerobic and musclestrengthening. An aerobic activity is that which gets ones heart beat faster and breathe harder than when one is not performing it. It includes activities from walking, running, pushing a lawn mower, to biking to the store etc. Musclestrengthening activities are those which work all the major muscle groups of the body (legs, hips, back, chest, abdomen, shoulders, and arms).

Each physical activity is defined by its duration and intensity. Duration is the amount of time spent participating in a physical activity session. Intensity is the rate of energy expenditure while performing physical activity. Intensity can be considered as either light, moderate or vigorous.

Walking is the most common and inexpensive form of the physical activity and has been shown to be an integral component of the physical activities performed by the adults's population[21]. According to CDC guidelines [4], it is recommended that adults should perform at least 150 minutes of moderate-intensity aerobic activity i.e. brisk walking per week or 30 minutes of walk five times a week to remain healthy and physically fit. Health benefits can also be achieved when this goal is completed as a series of shorter bouts i.e. 10 minutes of activity three time a day. However if we consider walk as the performed activity, then user cannot be categorized into any activeness level, only on the basis of activity duration. Every person has its own walking pace depending upon weight, age, height etc. Walking for 10 minutes is different for every person depending on his/her walking pace. Thus a person walking with the fast pace will cover more distance and perform more activity as compared to the one walking with slow pace. Let's take a person who performs 10 minutes of walk and take about 1000 steps while another person also performs 10 minutes of walk but take 800 steps. Both have performed same activity for same duration but the first one has performed more activity as well as covered more distance as compared to second one. This depicts that while walking step count should be considered for categorizing user into any activeness level.

Keeping this as our motivation we propose a framework that take walk as the performed activity and consider user's step count rather then activity duration in order to differentiate
TABLE I: Rules for Determining Activeness Level

\begin{tabular}{|l|l|}
\hline Activity State & Moderate \\
\hline Sedentary & NIL \\
\hline Lightly Active & Light exercise 1-3 days \\
\hline Mod. Active & total $>150 \mathrm{~min}$ \\
\hline Active & total $>200 \mathrm{~min}$ \\
\hline Energetic & total $>300 \mathrm{~min}$ \\
\hline
\end{tabular}

between activeness levels. These steps are then converted to the duration for which activity has been performed. Marshall et al. [22] performed a research for translating current physical activity recommendation to pedometer based guidelines. A sample of 97 adults consisting of both males and females with different ages, weights (Normal weight, overweight,obese) and height was taken. They proposed that moderate intensity walking appears approximately equal to at least 100 steps per minute for adults. To meet the goal of 30 minutes moderate intensity walk in a day, individuals have to walk at the speed of 100 steps per minute thus covering 3000 steps in 30 minutes and 15000 steps per week. We consider 100 steps per minutes as a threshold for converting user's steps into activity duration. Considering this, we derive a formula to convert user's step into duration of activity as:

$$
\text { walkingDuration }(\text { minutes })=\frac{\text { StepCount }}{100}
$$

This duration separates the users on the basis of their steps rather then duration. Now if a person performs 1000 steps in 10 minutes then by our formula his/her walkingDuration will be 10 minutes but if a person performs 800 steps in 10 minutes then as per our formula his/her walkingDuration will be 8 minutes rather then 10 minutes which is his/her actual duration.

Based on CDC guidelines [4] and 100 steps per minute threshold as specified by [22] we developed a categorization mechanism based on steps count for measuring walking activity in adults. Table I shows these rules. User's step count are converted into activity duration which is then used to categorize user into different activeness level. walkingDuration is used for categorization instead of step count as all of existing health recommendations [4], [20] describes activeness level with respect to duration for which the activity has been performed. By reflecting user' progress in the form of activeness levels rather then step count will ultimately help users to gauge their performed activities with the existing health recommendations. This idea sets a strong basis for our application and makes our solution distinct from existing systems.

\section{SMARTFIT}

SmartFit is an Android based game that uses sensor enabled mobile phones and tends to change adult's lifestyle by promoting physical activities. The complete workflow of SmartFit is shown in Figure 1. Users enter their goals by using the application interface. SmartFit works by counting user's steps with the help of sensors embedded in the smart phones. This count is then changed to duration for which activity has been performed, a concept portrayed under the section III. Points are awarded if users fulfill their goal while points are deducted if they lags behinds, a method described in detail in section V. 
User's total score is then converted into trophies and badges which is then displayed as feedback to user on the application's interface. The feedback is also presented in the form of user's daily, weekly and monthly progress. The objective is to keep users motivated to have a healthy life style as well as engage them for longer period. Beginning with prototype, SmartFit comprises of following components.

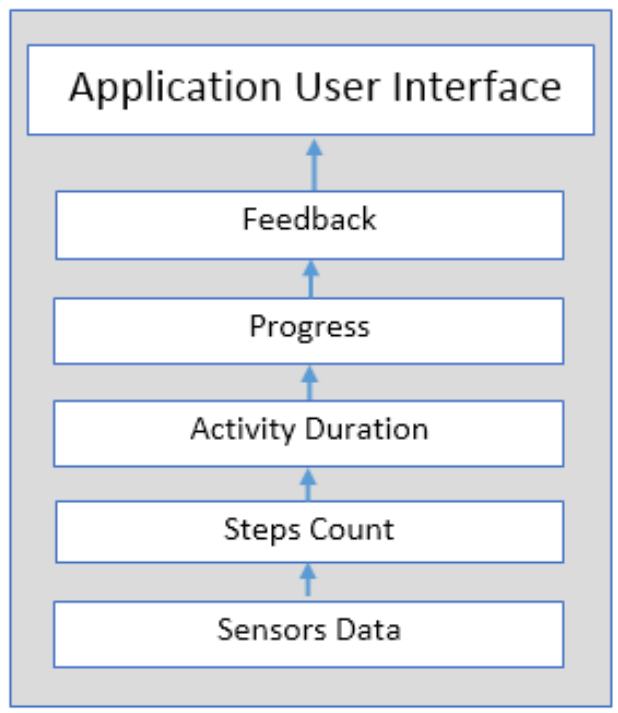

Fig. 1: Workflow for SmartFit

\section{A. Goal Initialization}

Users can have one of three goals: to be Moderately active, active or energetic. They set their goals at the start of the application but these goals can be customized in future as per needs. They will select their target level as how much active they want to see themselves in order to have a healthy life style. Goals setting theory [23] suggests that for changing the behavior the target goal must be broken into smaller goals that are easy to achieve rather then focusing on goals that are difficult to complete. Keeping this in mind, target level is divided into sub levels that user has to achieve on daily basis. These sub levels lead users ultimately to the final goal. Users will also be asked to enter the duration of exercise they would perform daily as their daily activity target. This daily activity target serves as the sub goal to the target goal. The daily activity target will be set at the start but can be changed lately. Figure 2 shows the SmartFit's interface for setting the goals.

\section{B. Activity Recognition}

SmartFit aims to continuously monitor user's behavior and detect number of steps when he/she has performed some physical activity. Till now we have only focused on walking activity. Google Fit ${ }^{2}$ is a health-tracking platform developed by Google for the Android operating system. Google Fit uses sensors in a user's mobile device to record physical fitness activities (such as step count, walking, running or cycling).

\footnotetext{
${ }^{2}$ https://developers.google.com/fit/overview
}

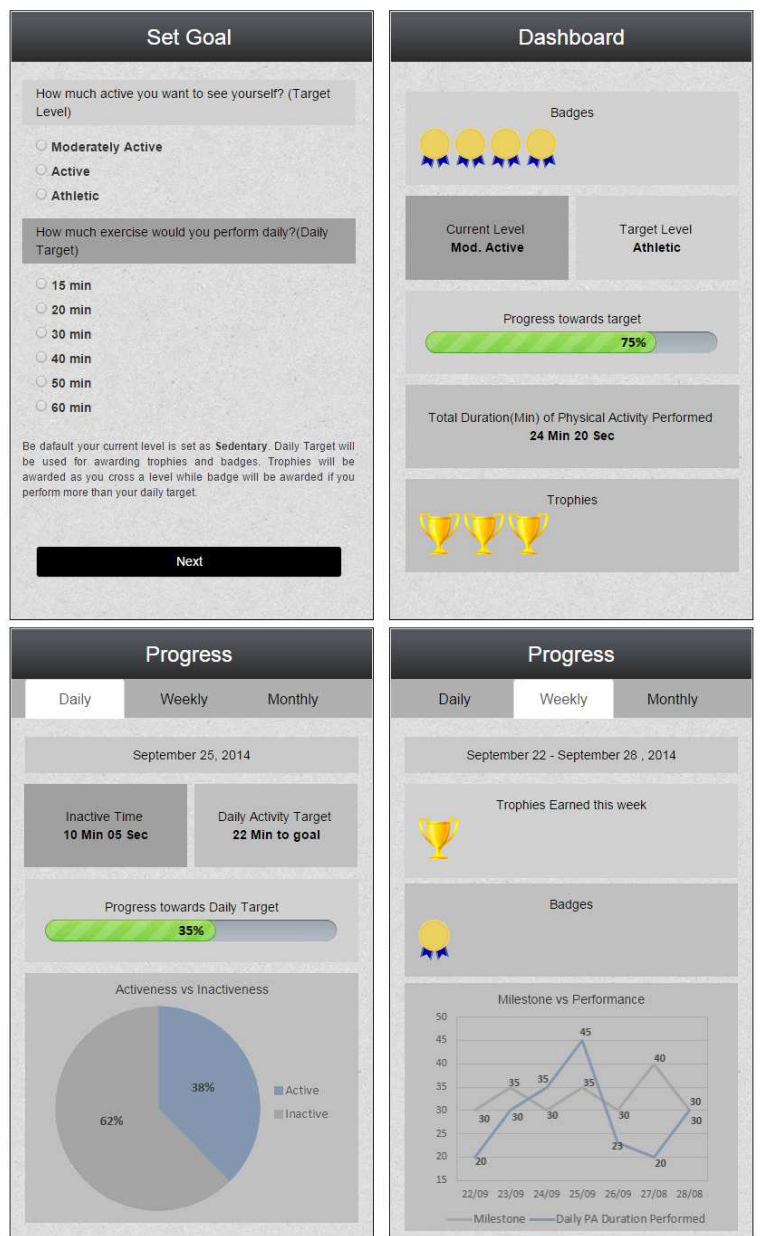

Fig. 2: a) Set Goals b) SmartFit Dashboard c) User's Daily Progress d) User's Weekly Progress

SmartFit makes use of Google Fit API to recognize number of steps taken by user. The objective of activity recognition is to identify user's behavior and then motivate him/her that in order to achieve the daily activity target, they have to do some physical activity.

\section{Progress Monitoring Dashboard}

Feedback is provided to users by reflecting their progress timely on SmartFit's dashboard. Users can monitor their progress by viewing the dashboard. The dashboard provides complete information about the goal. User's current progress towards achieving the final goal is shown in the form of progress bar on the dashboard and on mobile's notification bar. Dashboard also shows the points earned by the user in the form of trophies and badges as well as total duration of exercise performed by him/her. Users can also view their daily, weekly and monthly progress of activities performed by them. Daily progress shows the time period for which the user is inactive. The progress towards achieving the daily activity target is also shown in the form of progress bar. This allow SmartFit users to monitor their progress and how they can plan their actions for completing their daily target. Figure 2 shows the dashboard and daily, weekly progress screens of 
SmartFit.

\section{GAMIFICATION IN SMARTFit}

Incorporating gamification techniques in the health systems have been proven as the positive source of engagement for users. As stated by Baranowski, et al. [24], duration of physical activity may be increased by integrating fun enhancing procedures. Increasing difficulties with player skills, feedback on performance and awarding achievement badges increases user satisfaction which in turn results in increased physical activity. For calculating users progress, we have developed a scoring system in SmartFit that awards/deducts users points by the function of progress made by him/her. Users set their target level and perform physical activities in order to achieve the desired target. The point system is described as following.

\section{A. Rewards}

Users are awarded with points based on physical activities performed by them. If the user's performance is up to expectation then he/she is awarded with points while they are deducted if the user lags behind. Most of gamification techniques also focuses on using awards/badges as intermediate awards for rewarding users. SmartFit represents user's points in the form of trophies and badges. Badges are awarded when user crosses a level while trophies are awarded if the user performs more then the duration of daily activity target. If the user does not perform any activity for the longer period of time, then he/she is penalized and even downgraded to lower level which eventually results in the return of the badge.

\section{B. Levels}

SmartFit constitute of five different levels ranging from Sedentary to Energetic. User's initial level is set to sedentary and is updated as he/she gets promoted to higher levels. Users are promoted to higher levels on the basis of points they earned by performing activities. Figure 3 shows the five levels of the game. The minimum level is sedentary while the maximum level of activeness that user can achieve is Energetic. Each level is associated with a milestone that has to be achieved by user in order to cross that level. When user's points crosses his/her current level milestone then user will be promoted to the next level. However if a user's points falls below his/her current level milestone then he/she is downgraded to the lower level. The levels along with their corresponding milestone have been shown in Table II. Value of each level's milestone has been set after carrying out several experiments. These values are set in such a way that they translate the Table I levels into SmartFit's levels. The notion of levels gives the user a sense of accomplishment and progress toward meeting the larger goal.

\section{Point System}

Points are awarded to users in order to keep them engaged towards the application thus achieving the objective to keep users healthy and active. Points are not only awarded but also deducted by the function of progress made by user. The starting score is initialized to zero and is updated as the user progresses

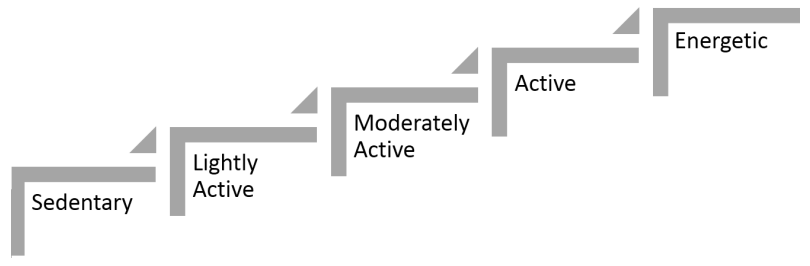

Fig. 3: Game Levels

TABLE II: Game Levels and Milestones for upgrade between levels

\begin{tabular}{|l|c|}
\hline Level & Milestone (Points) \\
\hline Sedentary & 30 \\
\hline Lightly Active & 70 \\
\hline Mod. Active & 130 \\
\hline Active & 220 \\
\hline Energetic & 380 \\
\hline
\end{tabular}

and performs some physical activities. Some of key points of SmartFit's points system are as follow:

- User provides daily activity target as his/her preference. Daily activity target is used to award/deduct points. This gives user freedom to perform exercise as per their preferences thus providing them control over the system.

- Steps are counted and converted to walking duration as shown in Equation (1).

- User's daily score is defined as:

$$
\text { dailyScore }=\text { walkingDuration }
$$

- If user's daily Score is less then daily activity target then we define penalty as:

$$
\text { penalty }=\text { dailyActivityTarget }- \text { dailyScore }
$$

However if a user completes daily activity target then penalty is zero as he/she completes the goal successfully so instead of penalizing, smartFit awards user with points.

1) Incorporating Endurance and Fallback: D. Wortley suggests that gamification works by setting goals, rewarding winners and penalizing losers [25]. In order to incorporate the penalizing part in SmartFit, we introduce factors of Endurance and Fallback. Endurance is associated with each level and its value is shown in Table III. The value of fallback is set to 0.3 and it is same for each level. The values of endurance and fallback has been set after carrying out several experiments. Endurance is used for penalizing user on daily basis. It is included in SmartFit for motivating the user that he/she has to perform recommended level of physical activities regularly. If the user underperforms as compared to his/her current level then due to endurance he/she will be immediately downgraded to lower level. Furthermore endurance also makes the completion of level challenging thus encouraging the user that he/she has to perform walk on daily basis in order to hold the current level.

Fallback is used for penalizing the user on weekly basis. It is introduced in SmartFit for keeping the user's performance consistent over the week. If user performance is found to 

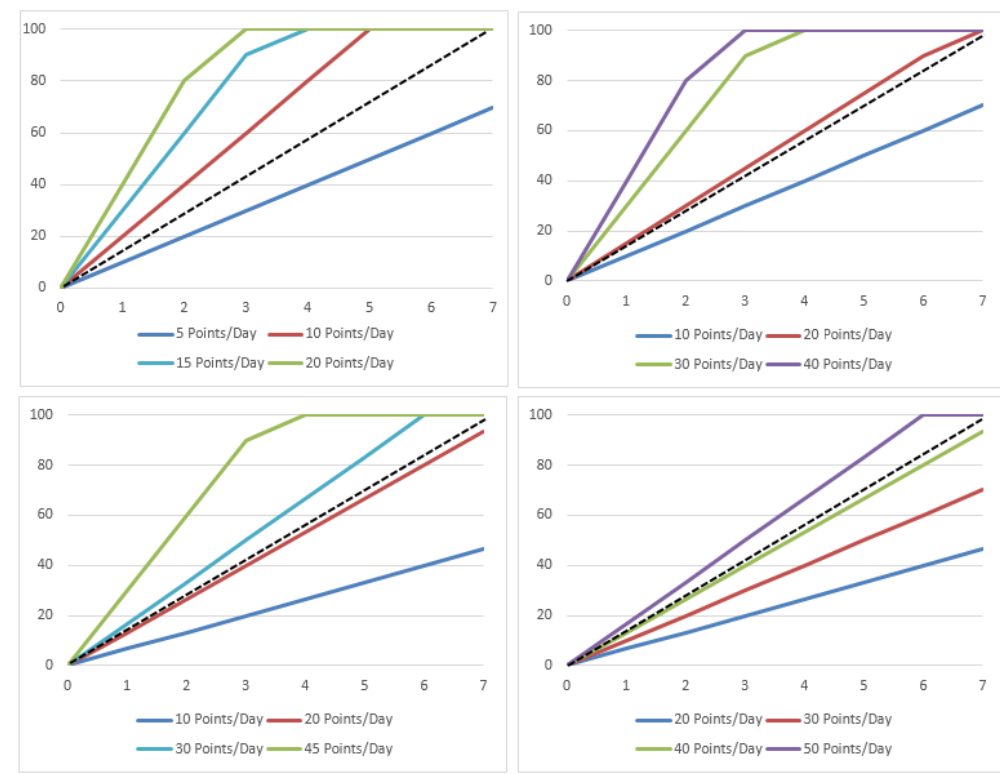

Fig. 4: Simulation Results for identifying level's milestone a)Lightly Active b)Moderately Active c)Active d)Energetic

TABLE III: Endurance for each Game Level

\begin{tabular}{|l|c|}
\hline Level & Endurance \\
\hline Sedentary & 0.7 \\
\hline Lightly Active & 0.8 \\
\hline Mod. Active & 0.8 \\
\hline Active & 0.8 \\
\hline Energetic & 0.8 \\
\hline
\end{tabular}

be unsatisfactory during the week then his/her level will be dropped. This keeps the user motivated that he/she has to perform consistently in order to hold the current level as well as for progressing towards higher levels. Also through experiments we get to know that penalizing users on weekly basis makes the point system balanced.

2) Score Calculation: Keeping in view the above criteria, user score is calculated by using the following algorithm.

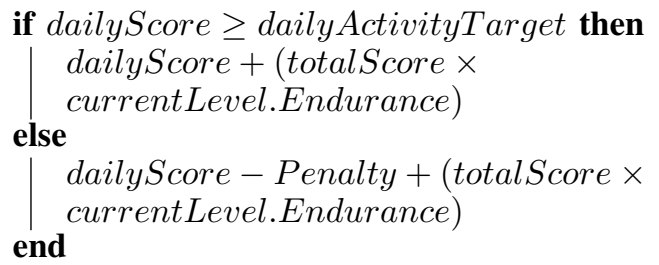

At the end of week, the user's points are calculated as:

$$
\text { totalScore }=\text { totalScore }-(\text { totalScore } * 0.3)
$$

\section{Simulation Results and Discussion}

The proposed point system was defined after carrying out several experiments in which values of level's milestone, endurance and fallback has been identified. These experiments are carried out with the intention to find out such values of milestone, endurance and fallback that keep the balance between user's skills and user engagement. This balance will serve the purpose of engaging the user for longer period. Furthemore values of level's milestone has been set in such a way that they translate the Table I levels into SmartFit's levels thus making both levels equivalent.

Figure 4 shows the results of experiments performed to determine the milestone of each activeness levels. Table I specifies the duration of exercise required in a week in order to reach a certain level. Thus we have performed the experiment for the time period of seven days so to convert per week duration into daily points. The dotted line in Figure 4 represents the optimal performance. User has to earn points that crosses this line and $\mathrm{keep} \mathrm{him/her} \mathrm{as} \mathrm{close} \mathrm{as} \mathrm{possible} \mathrm{to} \mathrm{this} \mathrm{line.} \mathrm{Experiments} \mathrm{have}$ been performed by taking different points per day and then the results are compared with the optimal line. If we consider Figure 4 (a) i.e. Lightly Active Level then we can see that the line representing 10 points per day crosses the optimal line as well as it is closest to that line. Thus the milestone for Lightly Active is set to 10 points per day. This means that user has to perform physical activity of 10 minutes on daily basis for maintaining the Lightly Active level. But if a user does not perform 10 points per day then he/she will be downgraded to the lower level which in this case is sedentary. Similarly to reach Moderately Active level user has to perform at least 20 minutes of exercise everyday as the line representing 20 points per day crosses the optimal line and is nearest to that line.

\section{A. Step Recognition Accuracy}

As described earlier, SmartFit makes use of Google Fit API for counting the steps taken by the user while walking. So it is necessary to first check the accuracy of step recognition of Google Fit. For this purpose we performed a experiment with 5 subjects, 2 female and 3 male. The subjects were asked 


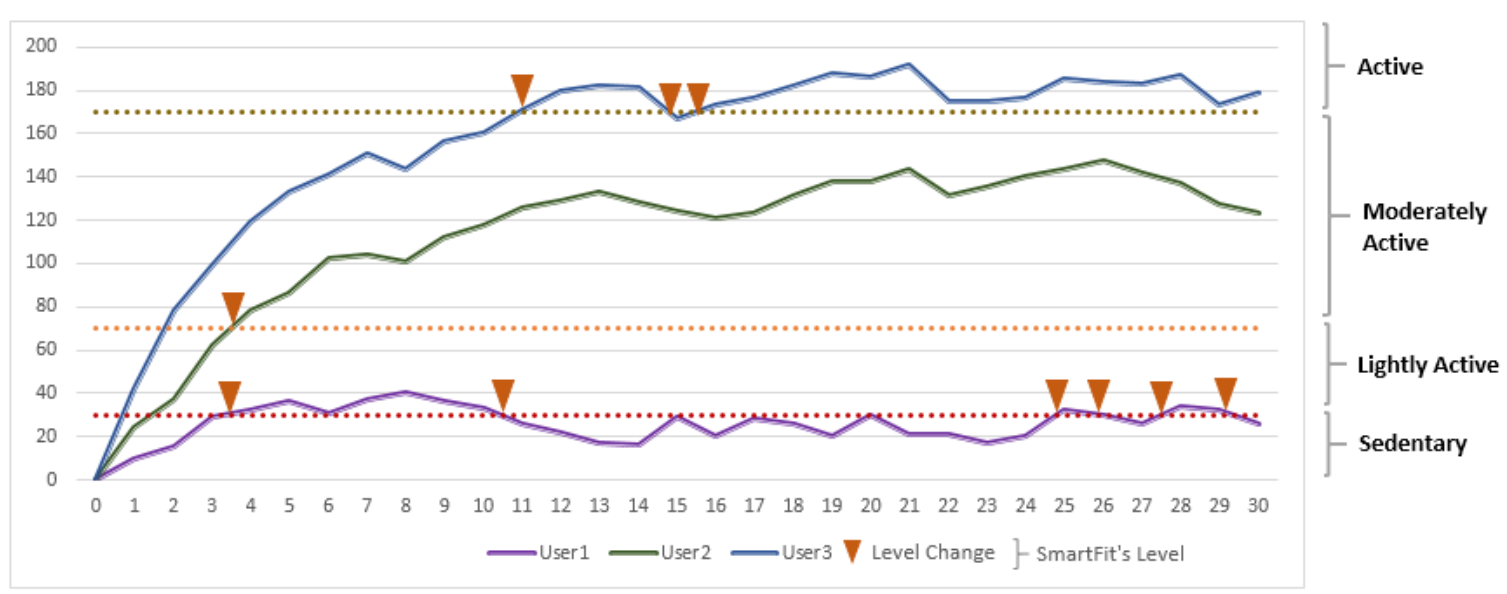

Fig. 5: User Performance vs. Level change

TABLE IV: Summary of Results obtained on testing Google Fit

\begin{tabular}{|l|c|c|c|}
\hline Gender & $\begin{array}{c}\text { Actual } \\
\text { Steps }\end{array}$ & $\begin{array}{c}\text { Detected } \\
\text { Steps }\end{array}$ & Accuracy \\
\hline Female & 100 & 92 & $92 \%$ \\
\hline Female & 200 & 185 & $93 \%$ \\
\hline Male & 150 & 145 & $97 \%$ \\
\hline Male & 220 & 171 & $78 \%$ \\
\hline Male & 243 & 172 & $71 \%$ \\
\hline
\end{tabular}

to count the number of steps taken by them. These steps are then compared with the steps detected by Google Fit so an assessment of accuracy could be made. Users were asked to carry the cell phone running the Google Fit in their hands. The results of the experiment are displayed in Table IV. The average accuracy of Google Fit turns out to be $86 \%$.

\section{B. SmartFit's Evaluation}

Now we will present simulation results of our prototype in order to evaluate that whether SmartFit places user into his/her activeness level as specified in Table I. Till now we have evaluated SmartFit on a small scale by considering three users. SmartFit's behavior was simulated for the period of 30 days and results are shown in Figure 5.

User1 takes on average 1500 steps (Min: 1000 , Max: 2000) daily, user2 takes 3000 steps (Min: 2500 , Max: 3500) on average and user3 takes 4000 steps (Min: 3500, Max: 4500) on average while walking. On converting user's step into walking duration then user1 performs 15 minutes of walk on average while user2 do 30 minutes of walk daily and user 3 do 40 minutes of walk on average daily. Daily activity target is set to 30 minutes for all the users. What we observe from these readings is that endurance and fallback continuously upgrades/downgrades user level by the function of his/her performance as well as makes the completion of level challenging, thus motivating the user to perform more in order to be active. According to Table III, milestone for Moderately Active level is more as compared to Lightly Active/ Sedentary level. Thus the graph rises very quickly for Moderately Active users while graph for Sedentary or Lightly Active users rises slowly. So the users targeting the higher levels have to perform more in order to reach their desired goal as compared to users targeting the lower levels.

As described above, 15 mins of walk is required daily in order to categorize user as Lightly Active while 30 mins are required to move towards Moderately Active level. User1 behavior has been identified as Lightly Active so SmartFit ensures that he never crosses Lightly Active level. Thus after 28 days he is on Lightly Active level. However variations can be seen between Sedentary and Lightly Active level for user1 in order to motivate him more towards performing activities.

User2 performs walk of on average 30 min of walk daily which is enough to declare him as Moderately Active. Thus SmartFit upgrades the user2's level from sedentary to Lightly Active in two days while level will be changed from Lightly Active to Moderately Active after 4 days and then SmartFit helps user to maintain this level.

User3 performs on average 40 mins of walk daily due to which SmartFit identifies her as Active and moves her quickly to the corresponding level. However variations will be seen between Moderately Active and Active level for User3. These variations are due to SmartFit's scoring system that motivates user that she has to perform more in order to be maintain Active Level. Now if we consider the performance of user 1 and user 3 on day 14 then we can see the benefit of using fallback factor. At day 14, the total score of user1 falls as his previous week performance is not up to the mark. User1 underperforms during the whole week and as the result points are deducted from his total score. This deduction of point is due to fallback factor and it is used to motivate the user to perform the recommended level of physical activity consistently. But if we see the performance of user 3 in 2 nd week i.e. from day 7 to day 14 then her performance is up to the mark. User3 has improved her score during the whole week so at the end of week the fraction of points that has been deducted from her score does not cause enough damage to user3's current level and score as compared to user1.

What we see from these simulations is that Smartfit's scoring system not only categorizes users in their respective activeness levels as specified in Table I but by deducting points they also keep them motivated to perform some exercise daily. SmartFit also upgrades/downgrades user's level depending on his/her progress so to keep him engaged and motivated for retaining 
the current level. However we need to test our system on real users to find out its impact on their behavior.

\section{CONCLUSION AND FUTURE WORK}

It is recommended that adults should perform 150 minutes of moderate intensity aerobic activity per week to remain healthy and physically fit. However when walk is considered as the performed activity, then activity duration is not enough for categorizing user into any activeness level. Step count is another factor that should be taken into account. Users can perform activity for same duration but they can differ depending on their step count. This article presents an approach for converting users step into the duration for which activity has been performed. We have developed a prototype, SmartFit that uses user step count and converts these steps to activity duration. The objective of SmartFit is to promote physical activities and enable users to achieve their activeness goal by dividing the goal into level and sublevels. Using gamification techniques, users are awarded with points if their performance is up to mark while points are deducted if a user lags behind in completing his/her goal. Unlike existing systems, which focus on the goal itself, our focus is on facilitating the user to achieve the goal.

To ensure that our approach is applicable to wide variety of users, we intend to conduct experiments on larger scale consisting users from different population, age groups, gender etc. After that we will be confident about the impact of SmartFit on user lifestyle.

Moreover, we plan to incorporate user preferences as well as exercise recommendation in SmartFit. User will enter his/her preferences e.g. weight, age, health condition etc. The exercise will be recommended by considering the user's preferences and points are deducted accordingly.

\section{REFERENCES}

[1] Genevieve N Healy, Charles E Matthews, David W Dunstan, Elisabeth AH Winkler, and Neville Owen. Sedentary time and cardiometabolic biomarkers in us adults: Nhanes 2003-06. European heart journal, page ehq451, 2011.

[2] Simon J Marshall and Ernesto Ramirez. Reducing sedentary behavior a new paradigm in physical activity promotion. American Journal of Lifestyle Medicine, 5(6):518-530, 2011.

[3] Michael Babyak, James A Blumenthal, Steve Herman, Parinda Khatri, Murali Doraiswamy, Kathleen Moore, W Edward Craighead, Teri T Baldewicz, and K Ranga Krishnan. Exercise treatment for major depression: maintenance of therapeutic benefit at 10 months. Psychosomatic medicine, 62(5):633-638, 2000.

[4] Centers for Disease Control and Prevention. Recommendations for physical activities, http://www.cdc.gov/physicalactivity/everyone/ guidelines/adults.html.

[5] Sebastian Deterding, Miguel Sicart, Lennart Nacke, Kenton O'Hara, and Dan Dixon. Gamification. using game-design elements in nongaming contexts. In CHI'11 Extended Abstracts on Human Factors in Computing Systems, pages 2425-2428. ACM, 2011.

[6] Jane McGonigal. Reality is broken: Why games make us better and how they can change the world. Penguin, 2011.

[7] Thijs Franck. How to engineer an app that changes habits effectively. 21st Twente Student Conference on IT, 2014.

[8] Brian J Fogg. Persuasive technology: using computers to change what we think and do. Ubiquity, 2002(December):5, 2002.

[9] Ian Li, Jodi Forlizzi, and Anind Dey. Know thyself: monitoring and reflecting on facets of one's life. In CHI'10 Extended Abstracts on Human Factors in Computing Systems, pages 4489-4492. ACM, 2010.
[10] Ian Li, Yevgeniy Medynskiy, Jon Froehlich, and Jakob Larsen. Personal informatics in practice: improving quality of life through data. In CHI'12 Extended Abstracts on Human Factors in Computing Systems, pages 2799-2802. ACM, 2012.

[11] Saskia Dantzig, Gijs Geleijnse, and Aart Tijmen Halteren. Toward a persuasive mobile application to reduce sedentary behavior. Personal and ubiquitous computing, 17(6):1237-1246, 2013.

[12] Laura R Pina, Ernesto Ramirez, and William G Griswold. Fitbit+: A behavior-based intervention system to reduce sedentary behavior. In Pervasive Computing Technologies for Healthcare (PervasiveHealth), 2012 6th International Conference on, pages 175-178. IEEE, 2012.

[13] Yuzhong Lin, Joran Jessurun, Bauke de Vries, and Harry Timmermans. Motivate: context aware mobile application for activity recommendation. In Ambient Intelligence, pages 210-214. Springer, 2011.

[14] Ashraf Khalil and Suha Glal. Stepup: a step counter mobile application to promote healthy lifestyle. In Current Trends in Information Technology (CTIT), 2009 International Conference on the, pages 1-5. IEEE, 2009.

[15] Nael Hirzallah. A simple exercise-to-play proposal that would reduce games addiction and keep players healthy. International Journal of Advanced Computer Science and Applications (IJACSA), 4(2), 2013.

[16] Hamid Mukhtar and Djamel Belaid. Using adaptive feedback for promoting awareness about physical activeness in adults. In Ubiquitous Intelligence and Computing, 2013 IEEE 10th International Conference on and 10th International Conference on Autonomic and Trusted Computing (UIC/ATC), pages 638-643. IEEE, 2013.

[17] Vijay Rajanna, Raniero Lara-Garduno, Dev Jyoti Behera, Karthic Madanagopal, Daniel Goldberg, and Tracy Hammond. Step up life: A context aware health assistant. 2014.

[18] Matthijs Jan Zwinderman, Azadeh Shirzad, Xinyu Ma, Prina Bajracharya, Hans Sandberg, and Maurits Clemens Kaptein. Phone row: a smartphone game designed to persuade people to engage in moderateintensity physical activity. In Persuasive Technology. Design for Health and Safety, pages 55-66. Springer, 2012.

[19] Pavol Bielik, Michal Tomlein, Peter Krátky, Štefan Mitrík, Michal Barla, and Mária Bieliková. Move2play: an innovative approach to encouraging people to be more physically active. In Proceedings of the 2nd ACM SIGHIT International Health Informatics Symposium, pages 61-70. ACM, 2012.

[20] World Health Organization. Global recommendations on physical activity for health. W.H.O. report, 2010, ISBN: 9789241599979.

[21] Thomas Stephens, David R Jacobs Jr, and Craig C White. A descriptive epidemiology of leisure-time physical activity. Public health reports, 100(2): 147, 1985.

[22] Simon J Marshall, Susan S Levy, Catrine E Tudor-Locke, Fred W Kolkhorst, Karen M Wooten, Ming Ji, Caroline A Macera, and Barbara E Ainsworth. Translating physical activity recommendations into a pedometer-based step goal: 3000 steps in 30 minutes. American journal of preventive medicine, 36(5):410-415, 2009.

[23] HA Schut and HJ Stam. Goals in rehabilitation teamwork. Disability \& Rehabilitation, 16(4):223-226, 1994.

[24] Tom Baranowski, Ralph Maddison, Ann Maloney, Ernie Medina Jr, and Monique Simons. Building a better mousetrap (exergame) to increase youth physical activity. GAMES FOR HEALTH: Research, Development, and Clinical Applications, 3(2):72-78, 2014.

[25] David Wortley. Gamification and geospatial health management. In IOP Conference Series: Earth and Environmental Science, volume 20, page 012039. IOP Publishing, 2014. 